\section{Association between Helicobacter pylori infection and Posner-Schlossman syndrome}

${ }^{1}$ Department of Ophthalmology, Sungkyunkwan University School of Medicine, Kangbuk Samsung Hospital, Seoul, Korea

${ }^{2}$ Department of Ophthalmology, Seoul National University College of Medicine, Seoul, Korea

${ }^{3}$ Dr Hong's Eye Clinic, Seoul, Korea

Correspondence: JM Kim, Department of Ophthalmology, Sungkyunkwan University School of Medicine, Kangbuk Samsung Hospital, Seoul,

Korea.

Tel: + 82220012257 ;

Fax: + 82220012262.

E-mail: kjoonmo@

dreamwiz.com

Received: 17 September 2008

Accepted in revised form: 24 January 2009

Published online: 20

February 2009

None of the authors have financial or proprietary interest in any of the materials mentioned

\begin{abstract}
Objective: To investigate possible association between Helicobacter pylori infection and Posner-Schlossman syndrome.

Methods: In a prospective study, 40 subjects with Posner-Schlossman syndrome were examined. As a control group, 73 subjects without Posner-Schlossman syndrome were selected for comparison with the study group. All participants underwent serologic analysis for the presence of H. pylori infection by an enzyme-linked immunosorbent assay. Positive rate of serum anti-H. pylori IgG was compared between Posner-Schlossman syndrome patients and control participants. Results: The rate of positive titre $(>15 \mathrm{U} / \mathrm{ml}$ ) of $H$. pylori was $80.0 \%$ in the Posner-Schlossman syndrome group and 56.2\% in control group, showing significant difference (Fischer's exact, $P=0.014)$. Multivariate analysis adjusted for various systemic factors revealed that positive titre of anti-H. pylori IgG was a significant risk factor for Posner-Schlossman syndrome with an odds ratio, $(4.84,95.0 \%$ CI: 1.10-21.24, $P=0.037$ ).

Conclusions: H. pylori infection occurred significantly more often in PosnerSchlossman syndrome patients. This study suggests that exposure to $H$. pylori infection is associated with Posner-Schlossman syndrome in Korea.

Eye (2010) 24, 64-69; doi:10.1038/eye.2009.34; published online 20 February 2009
\end{abstract}

Keywords: Posner-Schlossman syndrome; glaucomatocyclitic crisis; Helicobacter pylori; anterior uveitis

\section{Introduction}

Posner-Schlossman syndrome (PSS), also known as glaucomatocyclitic crisis, is an
CY Choi', MS Kim', JM Kim', SH Park'1, KH Park ${ }^{2}$ and $\mathrm{C} \mathrm{Hong}^{3}$ uncommon unilateral syndrome with recurrent attacks of anterior non-granulomatous uveitis and elevated intraocular pressure (IOP). With acute attack, IOP is typically $40-60 \mathrm{mmHg}$. After an acute attack, IOP and facility of aqueous outflow return to normal. ${ }^{1}$ It has been considered as a benign, self-limited disease because short-term use of topical corticosteroids and antiglaucoma medications usually control the attacks. But the aetiology of PSS remains still unknown, so there have been numerous postulations for the pathogenesis of PSS including abnormal vascular reactivity, autonomic dysregulation, and infections. Herpes simplex virus or cytomegalovirus has been suggested as aetiologies for PSS due to their presence in the aqueous humour during the acute attacks. ${ }^{2,3}$ Genetic factors, such as HLA-Bw54, was also considered for the aetiology. ${ }^{4}$

Helicobacter pylori, a curved spiral Gramnegative bacterium that colonies in the gastric mucosa, is one of the most common infections in human involving about half of human population. ${ }^{5}$ Prevalence of $H$. pylori infection is known to be differed between developing countries and developed countries, and according to ethnicity, place of birth, and socioeconomic factors among people living in the same country. ${ }^{6}$ In Korea, 56.0\% of 15916 population-based subjects were positive for anti-H. pylori IgG infection in 2005, and the seroprevalence of asymptomatic healthy subjects were significantly decreased in comparison to those in 1998 (59.6 vs 66.9\%). ${ }^{7,8}$

Knox ${ }^{9}$ has reported that PSS is associated with systemic disorders including allergic disorders and peptic ulcer disease. In that study, 32 PSS patients were evaluated and peptic ulcer was or had been shown in $68 \%$ of men and $38 \%$ of women in contrast to the fact that $10-20 \%$ of men and 5\% of women of western cultures have ulcers at sometime in their lives. Furthermore, 
the age distribution of the PSS patients was similar to that reported for peptic ulcer disease. Knox ${ }^{9}$ explained the association between PSS and peptic ulcer by following mechanism. Inflammation in the wall of an ulcer produce active cells and circulating antibodies or immune complexes that have the capacity to target on or cross-react with ocular tissue which is either immunologically similar or has become immunologically sensitized.

Peptic ulcer disease known to be related to PSS, ${ }^{9}$ and close association between $H$. pylori and gastric ulcer disease warrant an investigation about a link between PSS and H. pylori. The aim of this study is to reveal whether $H$. pylori infection is associated with PSS.

\section{Materials and methods}

Study subjects were recruited from 1 March 2006 to 31 December 2007 at the Department of Ophthalmology, Sungkyunkwan University School medicine, Kangbuk Samsung hospital, Seoul, Korea. An ethics committee approval was obtained from the Institutional Review Board. Participants were enrolled after the informed consent. Two groups were employed to investigate the association between $H$. pylori and PSS.

Group 1 consisted of consecutive 40 PSS patients diagnosed by ophthalmic examinations, including a slitlamp examination, IOP measurement with a Goldman applanation tonometer, and fundus and optic disc examinations using a $90 \mathrm{D}$ biomicropscopy. All patients had recurrent monocular anterior chamber inflammation at the unilateral affected eye. The inflammation was usually mild, with $1+$ anterior chamber cells and trace flare. Keratic precipatates also accompanied the anterior chamber inflammation in almost all subjects. The IOP exceeded $27 \mathrm{mmHg}$ at the initial visit or during the follow-up. Gonioscopy revealed open-angle and no peripherial anterior synichiae. All patients underwent one or more visual field examinations using a Humphrey visual field analyser (Carl zeiss meditec, Dubin, CA, USA). There was no evidence of visual field defect and optic disc abnormality indicating glaucoma at the initial visit.

In group 2, control participants, consisted of consecutive 254 patients who underwent $H$. pylori serologic test during the same study period as group 1 . All group 2 participants performed eyelid examinations for oculoplastic complaints or cataract assessment at our clinic. Subjects with glaucoma, previous ocular surgery, and serious external or retinal disease were excluded from the group 2. Patients aged 65 years or more were also excluded because there was no subject older than 65 years of age in the group 1. Seventy-three subjects were enrolled in this study after applying exclusion criteria.
Demographic data including age, gender, and previous history of diabetes mellitus and hypertension were obtained for the study. In group 1, best-corrected visual acuity (BCVA) and IOP with a Goldman applanation tonometer of the affected eye at the initial visit were recorded. In group 2, BCVA, IOP of right eye, and the $H$. pylori serologic test value were recorded. If cataract involved both eyes, BCVA and IOP of less affected eye were obtained for the analysis.

For laboratory tests of $\mathrm{H}$. pylori, venous samples were obtained from each subject. Anti-H. pylori immunoglobulin G (IgG) was determined quantitatively using an enzyme-linked immunosorbent assay (ELISA; Genedia H. pylori ELISA; Green Cross Medical Science Corp, Seoul, South Korea). The manufacturer's recommended cutoff value was $15 \mathrm{U} / \mathrm{ml}$ for $H$. pylori $\mathrm{IgG}$ to determine serologic status as positive or negative. PSS patients and control participants were tested by the same laboratory.

For statistical analysis, Mann-Whitney $U$-test was used for comparison of age, IOP, and BCVA between the two groups. Fisher's exact test was used for comparing gender, existence of diabetes mellitus and hypertension, and positive rate of the serologic test. Odds ratio between positivity of the serologic test and PSS was calculated with univariate and multivariate logistic regression. In multivariate analysis, age, gender, diabetes mellitus, and hypertension were adjusted factors. One sample $t$-test was used for the comparison between the positivity of the serologic test in the PSS group and age-matched expected rate of positivity calculated from the nationwide survey performed in $2005 .^{8}$

\section{Results}

The mean age of PSS patients in the group 1 was $43.8 \pm 13.0$ years. Twenty-six patients were men and 14 were women. The mean IOP of involved eye at the initial visit was $40.3 \pm 12.3 \mathrm{mmHg}$. There were no significant differences in the mean age, gender, logMAR of BCVA, and diabetes mellitus between groups 1 and 2 (Table 1). The mean IOP was significantly greater in the group 1 $(P<0.001)$, and group 2 subjects had more hypertension $(P=0.016)$. The rate of positive titre $(>15 \mathrm{U} / \mathrm{ml})$ of H. pylori was 80.0 and $56.2 \%$ in PSS and control groups, respectively, showing a significant difference (Fischer's exact, $P=0.014$; Table 1 ).

Table 2 shows risk factors for PSS. In univariate analysis, positive titre of anti-H. pylori IgG was a significant risk factor for PSS. After adjustment for age, gender, diabetes mellitus, hypertension, $\mathrm{HbsAg}$, and anti-Hbs IgG, positive titre of anti-H. pylori IgG was still a significant risk factor for PSS. 
Table 1 Clinical charateristics of PSS and non-PSS groups

\begin{tabular}{lccr}
\hline & Group 1 (PSS; $\mathrm{n}=40)$ & Group 2 (non-PSS; $\mathrm{n}=73)$ & P-value \\
\hline Mean age at diagnosis (years) & $43.8 \pm 13.0$ & $46.1 \pm 15.0$ & $0.423^{\mathrm{a}}$ \\
Male (\%) & 65.0 & 69.9 & $0.674^{\mathrm{b}}$ \\
Intraocular pressure (mmHg) & $40.3 \pm 12.3$ & $13.9 \pm 2.7$ & $0^{\mathrm{a}}$ \\
LogMAR of BCVA & $0.22 \pm 0.44$ & $0.13 \pm 0.31$ & $0.255^{\mathrm{a}}$ \\
Diabetes mellitus (\%) & 6.3 & 21.2 & $0.081^{\mathrm{b}}$ \\
Hypertension (\%) & 6.3 & 27.3 & $0.016^{\mathrm{b}}$ \\
HbsAg (\%) & 4.8 & 13.7 & $0.423^{\mathrm{b}}$ \\
Anti-HbsAb (\%) & 76.2 & 51.0 & $0.066^{\mathrm{b}}$ \\
Positive anti-H. pyori IgG titre (\%) & 80.0 & 56.2 & $0.014^{\mathrm{b}}$ \\
\hline
\end{tabular}

BCVA, best-corrected visual acuity; H. pyori, Helicobacter pylori; IgG, immunoglobulin; PSS, Ponser-Schlossman syndrome.

a Mann-whitney $U$-test.

${ }^{\mathrm{b}}$ Fischer's exact test.

Table 2 Univariate and multivariate analyses of risk factors for Posner-Schlossman syndrome

\begin{tabular}{|c|c|c|c|c|c|c|c|c|c|c|}
\hline \multirow[t]{2}{*}{ Variables } & \multicolumn{5}{|c|}{ Univairate analysis } & \multicolumn{5}{|c|}{ Multivariate analysis } \\
\hline & Odds ratio & & $95 \%$ & & P-value ${ }^{\mathrm{a}}$ & Odds ratio & & $95 \%$ & & P-value ${ }^{\mathrm{a}}$ \\
\hline Age (per 10 years) & 0.90 & 0.70 & to & 1.17 & 0.430 & 0.72 & 0.45 & to & 1.14 & 0.160 \\
\hline Male & 0.80 & 0.35 & to & 1.82 & 0.596 & 1.22 & 0.32 & to & 4.64 & 0.769 \\
\hline Diabetes mellitus & 0.25 & 0.05 & to & 1.16 & 0.077 & 0.30 & 0.06 & to & 1.49 & 0.141 \\
\hline Hypertension & 0.18 & 0.04 & to & 0.82 & 0.027 & 0.15 & 0.01 & to & 1.60 & 0.116 \\
\hline $\mathrm{HbsAg}$ & 0.31 & 0.04 & to & 2.73 & 0.294 & 0.55 & 0.03 & to & 9.96 & 0.688 \\
\hline Anti-Hbs IgG & 3.08 & 0.98 & to & 9.66 & 0.054 & 2.51 & 0.56 & to & 11.26 & 0.228 \\
\hline Anti-H. pylori IgG & 3.12 & 1.27 & to & 7.70 & 0.013 & 4.84 & 1.10 & to & 21.24 & 0.037 \\
\hline
\end{tabular}

CI, confidence interval; IgG, immunoglobulin.

aBinary logistic regression.

Table 3 Positive results of laboratory tests in group $1(n=40)$

\begin{tabular}{lc}
\hline Laboratory test & Number of patients \\
\hline Anti-varicella zoster virus IgG & 19 \\
Anti-herpes simplex virus IgG & 10 \\
HLA B27 & 3 \\
Anti-nuclear antibody & 1 \\
Rheumatoid factor & 1 \\
Anti-toxoplasma IgM & 1 \\
HIV & 0 \\
RPR & 0
\end{tabular}

IgG, immunoglobulin; RPR, rapid plasma reagin.

Table 3 showed laboratory tests for screening aetiology of uveitis in group 1. Anti-varicella zoster virus IgG, antiherpes simplex virus IgG, and HLA B27 were the three most frequent positive laboratory tests.

The expected rate of positive titre of anti-H. pylori IgG in a population with the same age distribution as groups 1 and 2 was calculated on the basis of the seroprevalence ( $>15 \mathrm{U} / \mathrm{ml}$ ) survey of $H$. pylori of Korean population conducted in 2005. The age-matched expected positive rate of group 1 was significantly lower than that of the current study $(51.5 \pm 12.8$ vs $80.0 \%, P<0.001)$, and the expected rate of group 2 showed no significant difference from that of our study $(52.6 \pm 13.8$ vs $56.2 \%, P=0.107)$.

\section{Discussion}

In this study, the positive rate of $H$. pylori infection was significantly higher in the PSS patients $(80.0 \%)$ than in the control patients $(56.2 \%)$ and even than the age-matched expected rate in the population of the national survey $(51.5 \pm 12.8 \%)$. The multivariate analysis-adjusted systemic factors also showed significant odds ratio (4.84, 95\% CI: 1.10-21.24; Table 2). The seropositivity of $H$. pylori in the observed rate (56.2\%) of the control group was not significantly different from the expected rate $(52.6 \pm 13.8 \%)$ calculated from the population-based seroprevalence survey in Korea. This similarity of seropositivity in the control group between observed and age-matched rates may be due to similar study period, that is, 2006-2007 vs 2005; as prevalence of $H$. pylori has been known to decrease as socioeconomic conditions improve, that also occurred in the Korean population. ${ }^{6-8}$

There are various diagnostic methods for H. pylori infection including invasive tests and non-invaisive tests. Invasive test are endoscopy, culture, molecular methods, 
urease test, and histology. Non-invasive tests are urea breath test, stool antigen test, PCR in stools, and antibody detection. ${ }^{10}$ These tests have different sensitivities and specificities. For gastroenterologists, the standard of diagnosis of $H$. pylori infection is endoscopic mucosal biopsy. Recently, endoscopy has become a safe diagnostic tool, although this procedure is not easy to perform in healthy asymptomatic individuals as it has still several potential risks for gastric adverse reaction and is relatively expensive. ELISA, used in this study, requires non-invasive venous sampling and detects previous H. pylori infection measuring anti-H. pylori IgG. The kit in this study used $H$. pylori antigen obtained from Korean H. pylori strains, and had a sensitivity and specificity of 97.8 and $92.0 \%$, respectively, in Korean adults. ${ }^{11}$ These sensitivity and specificity are not worse, but even better than those of other recent non-invasive methods. ${ }^{10}$ A limitation of this method is that it does not discriminate between current and old infection as it is a serology test. ${ }^{12}$ But, although the endoscopic biopsy and urea breath test are useful for active GI infection, they are not completely reliable for nondigestive disease. Furthermore, not only $H$. pylori itself may affect the ocular tissue, but also circulating antibody or immune complex can play a role to cause PSS. ${ }^{9}$ Thus, we chose ELISA for evaluation of the effect of $H$. pylori infection.

H. pylori induces a chronic atrophy in the gastric mucosa by autoantibody to $\mathrm{H}^{+}, \mathrm{K}^{+}$-ATPase that has been known to be the only mechanism reported as a pathway in trabecular meshwork. ${ }^{13}$ Pseudoexfoliation specimen was reported to share common histopathologic features associated with $H$. pylori-infected gastric ulcers. ${ }^{14}$ Relations of other ophthalmic diseases, such as central serous chorioretinopathy, ${ }^{15}$ glaucoma, ${ }^{16-20}$ acute anterior uveitis, ${ }^{21}$ and blepharitis, ${ }^{22}$ to $H$. pylori were reported recently in some literatures. $H$. pylori may induce ophthalmic disease through the following mechanisms:

(1) Promoting platelet aggregation and platelet-leukocyte aggregation. ${ }^{20}$

(2) Releasing proinflammatory and vasoactive substances, such as cytokines (IL-1, IL-6, IL-8, IL-10, IL-12, tumour necrosis factor (TNF)- $\alpha$, interferon (IFN)- $\gamma$ ), eicosanoids (leukotrienes, prostaglandins), and acute phase proteins (fibrinogen, C-reactive protein), increased endothelin-1 (a potent constrictor of arteioles and venules), nitric oxide, and inhibitor of nitric oxide synthase. ${ }^{20}$

(3) Induction of apoptosis. ${ }^{23}$

(4) Stimulating monocoagulant cell to induce a tissue factor-like procoagulant activity that converts fibrinogen into fibrin. ${ }^{24}$
(5) Causing the development of cross mimicry with endothelial antigen and Lewis- $x$ antigen. ${ }^{20,25}$

(6) Producing reactive oxygen metabolites and circulating lipid peroxides. ${ }^{20}$

(7) Induction of $H$. pylori heat-shock protein. ${ }^{26}$

(8) From the oral cavity, it may reach the anterior surface of the eye. ${ }^{22}$

Otasevic et $a l^{21}$ showed that the incidence of serum anti-H. pylori antibodies in acute anterior uveitis patients was significantly high, and they showed anti-H. pylori antibodies in the aqueous humour of acute anterior uveitis. In an ileocolonoscopic studies, $66 \%$ of acute anterior uveitis patients have gut inflammation. ${ }^{27}$ Impaired intestinal mucosa could allow the penetration of pathogenic bacterium into tissue provoking an immune reaction, and bacterium reach the eye by circulation. The same scenario could also take place in the stomach. ${ }^{21}$

Granfors $^{28}$ also showed that Gram-negative bacteria share uniquely high-sequence homologies with the HLA-B27 molecule. Antigenic similarities between Gram-negative bacteria and human component, such as epithelium of ciliary body, could develop immune reaction. As mentioned earlier, $H$. pylori antigen has cross mimicry with endothelial antigen and Lewis blood group antigen (Lewis $x$ ). ${ }^{25}$ Lewis- $x$ antigen is also present in the eye, and an autoimmune reaction may also be a possible pathway in PSS. $^{29}$

Besides the autoimmune reaction during $H$. pylori infection, $H$. pylori infection induces humoral and cellular immune response. The cellular response by H. pylori is Th- 1 cell response, and these cells produce IFN- $\gamma$ and TNF- $\alpha .{ }^{30}$ The activated macrophage by H. pylori also produce proinflammatory cytokines; IL-1, IL-6, IL-8, IL-10, IL-12, TNF- $\alpha$, and IFN- $\gamma \cdot{ }^{20}$ Most of these cytokines are also increased in the aqueous humour of the patients with idiopathic acute anterior uveitis, and TNF- $\alpha$ may participate actively in the pathogenesis of clinical uveitis. $^{31}$

H. pylori and its antibodies circulate the blood stream, and eventually enter the anterior chamber of both eyes through blood-aqueous humour barrier. However, PSS usually occurs repeatedly in unilateral eye, but not in bilateral eyes. This may be due to the fact that although H. pylori and its antibodies exist in the bilateral eyes, acute anterior uveitis that may initiate PSS occurs in the unilateral eye. The reason why the acute anterior uveitis occurs in mainly unilateral eye is still unknown, but we can postulate that the triggering effect of acute anterior chamber inflammation results in IOP rise by altering the outflow facility of aqueous humour by the coexistence of $H$. pylori infection. This hypothesis indicates that $H$. pylori may be an aggravating factor rather than a main 
aetiologic factor to cause PSS; however, verification of this hypothesis would be difficult because it may require a biopsy for the histological proof.

A limitation of this study is a small sample size. As PSS is an uncommon disease, the multicentred trials may be helpful to perform a study with greater number of subjects. In this study, hypertension seemed to be a protective factor for PSS in the univariate analysis. Hypertension is a very common systemic disease and small sample size of the current study may have lead to this result. Therefore, we performed multivariate analysis to overcome this weakness, and hypertension was not a significant protective factor for PSS in the multivariate analysis.

In conclusion, this study indicated that the prevalence of H. pylori infection in PSS patients was significantly higher than normal control group. After adjusting systemic factors, $H$. pylori was still a significant risk factor for the development of PSS. To elucidate how much H. pylori participate in the development of PSS, future studies should include comparison between acute unilateral anterior uveitis patients without increasing IOP and PSS patients. That may reveal the contribution of H. pylori to the occurrence of PSS more clearly by adjusting various factors for acute anterior uveitis.

\section{References}

1 Posner A, Schlossman A. Syndrome of unilateral recurrent attacks of glaucoma with cyclitic symptoms. Arch Ophthal 1948; 39(4): 517-535.

2 Yamamoto S, Pavan-Langston D, Tada R, Yamamoto R, Kinoshita S, Nishida $\mathrm{K}$ et al Possible role of herpes simplex virus in the origin of Posner-Schlossman syndrome. Am J Ophthalmol 1995; 119(6): 796-798.

3 Teoh SB, Thean L, Koay E. Cytomegalovirus in aetiology of Posner-Schlossman syndrome: evidence from quantitative polymerase chain reaction. Eye 2005; 19(12): 1338-1340.

4 Puri P, Verma D. Bilateral glaucomatocyclitic crisis in a patient with Holmes Adie syndrome. J Postgrad Med 1998; 44(3): 76-77.

5 NIH Consensus Conference. Helicobacter pylori in peptic ulcer disease. NIH Consensus Development Panel on Helicobacter pylori in Peptic Ulcer Disease. JAMA 1994; 272(1): 65-69.

6 Perez-Perez GI, Rothenbacher D, Brenner H. Epidemiology of Helicobacter pylori infection. Helicobacter 2004; 9(Suppl 1): 1-6.

7 Kim N. Epidemiology and transmission route of Helicobacter pylori infection. Korean J Gastroenterol 2005; 46(3): 153-158.

8 Yim JY, Kim N, Choi SH, Kim YS, Cho KR, Kim SS et al. Seroprevalence of Helicobacter pylori in South Korea. Helicobacter 2007; 12(4): 333-340.

9 Knox DL. Glaucomatocyclitic crises and systemic disease: peptic ulcer, other gastrointestinal disorders, allergy and stress. Trans Am Ophthalmol Soc 1988; 86: 473-495.
10 Granstrom M, Lehours P, Bengtsson C, Megraud F. Diagnosis of Helicobacter pylori. Helicobacter 2008; 13 (Suppl 1): 7-12.

11 Kim SY, Ahn JS, Ha YJ, Doh HJ, Jang MH, Chung SI et al. Serodiagnosis of Helicobacter pylori infection in Korean patients using enzyme-linked immunosorbent assay. J Immunoassay 1998; 19(4): 251-270.

12 Kountouras J, Zavos C, Katsinelos P, Grigoriadis N, Deretzi G, Tzilves D. Greek and Israeli patterns of Helicobacter pylori infection and their association with glaucoma: similarities or diversities? J Glaucoma 2008; 17(6): 503-504, author reply 506-507).

13 Claeys D, Faller G, Appelmelk BJ, Negrini R, Kirchner T. The gastric $\mathrm{H}+, \mathrm{K}+$-ATPase is a major autoantigen in chronic Helicobacter pylori gastritis with body mucosa atrophy. Gastroenterology 1998; 115(2): 340-347.

14 Bode G, Malfertheiner P, Mader U, Stanescu A, Ditschuneit $\mathrm{H}$. Fine structure of active and healed duodenal ulcer. Am J Gastroenterol 1991; 86(2): 179-186.

15 Giusti C, Mauget-Faysse M. Helicobacter pylori and idiopathic central serous chorioretinopathy. Swiss Med Wkly 2004; 134(27-28): 395-398.

16 Deshpande N, Lalitha P, Krishna das SR, Jethani J, Pillai RM, Robin A et al. Helicobacter pylori IgG antibodies in aqueous humor and serum of subjects with primary open angle and pseudo-exfoliation glaucoma in a South Indian population. J Glaucoma 2008; 17(8): 605-610.

17 Kountouras J, Zavos C, Grigoriadis N, Deretzi G, Katsinelos P, Tzilves D. Helicobacter pylori infection as an environmental familial clustering risk factor for primary open-angle glaucoma. Clin Experiment Ophthalmol 2008; 36(3): 296-297 author reply 297.

18 Kountouras J, Zavos C, Grigoriadis N, Deretzi G, Katsinelos P, Tzilves D. Helicobacter pylori infection as a risk factor for primary open-angle glaucoma. Clin Experiment Ophthalmol 2008; 36(2): 196.

19 Kountouras J, Mylopoulos N, Chatzopoulos D, Zavos C, Boura P, Konstas AG et al. Eradication of Helicobacter pylori may be beneficial in the management of chronic open-angle glaucoma. Arch Intern Med 2002; 162(11): $1237-1244$.

20 Kountouras J, Mylopoulos N, Boura P, Bessas C, Chatzopoulos D, Venizelos J et al. Relationship between Helicobacter pylori infection and glaucoma. Ophthalmology 2001; 108(3): 599-604.

21 Otasevic L, Walduck A, Meyer TF, Aebischer T, Hartmann $\mathrm{C}$, Orlic $\mathrm{N}$ et al. Helicobacter pylori infection in anterior uveitis. Infection 2005; 33(2): 82-85.

22 Sacca SC, Pascotto A, Venturino GM, Prigione G, Mastromarino A, Baldi F et al. Prevalence and treatment of Helicobacter pylori in patients with blepharitis. Invest Ophthalmol Vis Sci 2006; 47(2): 501-508.

23 Kountouras J, Zavos C, Chatzopoulos D. Induction of apoptosis as a proposed pathophysiological link between glaucoma and Helicobacter pylori infection. Med Hypotheses 2004; 62(3): 378-381.

24 Miragliotta G, Del Prete R, Mosca A. Helicobacter pylori infection and coronary heart disease. Lancet 1994; 344(8924): 751.

25 Appelmelk BJ, Simoons-Smit I, Negrini R, Moran AP, Aspinall GO, Forte JG et al. Potential role of molecular mimicry between Helicobacter pylori lipopolysaccharide and host Lewis blood group antigens in autoimmunity. Infect Immun 1996; 64(6): 2031-2040. 
26 Gobert AP, Bambou JC, Werts C, Balloy V, Chignard M, Moran AP et al. Helicobacter pylori heat-shock protein 60 mediates interleukin- 6 production by macrophages via a toll-like receptor (TLR)-2-, TLR-4-, and myeloid differentiation factor 88-independent mechanism. J Biol Chem 2004; 279(1): 245-250.

27 Mielants H, Veys EM, Cuvelier C, De Vos M. Course of gut inflammation in spondylarthropathies and therapeutic consequences. Baillieres Clin Rheumatol 1996; 10(1): 147-164.

28 Granfors K. Host-microbe interaction in reactive arthritis: does HLA-B27 have a direct effect? J Rheumatol 1998; 25(9): 1659-1661.
29 Ogiso M, Takehana M, Kobayashi S, Hoshi M. Expression of sialylated Lewisx gangliosides in cultured lens epithelial cells from rhesus monkey. Exp Eye Res 1998; 66(6): 765-773.

30 D'Elios MM, Manghetti M, De Carli M, Costa F, Baldari CT, Burroni $\mathrm{D}$ et al. T helper 1 effector cells specific for Helicobacter pylori in the gastric antrum of patients with peptic ulcer disease. J Immunol 1997; 158(2): 962-967.

31 Perez-Guijo V, Santos-Lacomba M, Sanchez-Hernandez M, Castro-Villegas Mdel C, Gallardo-Galera JM, CollantesEstevez E. Tumour necrosis factor-alpha levels in aqueous humour and serum from patients with uveitis: the involvement of HLA-B27. Curr Med Res Opin 2004; 20(2): 155-157. 